\title{
The Summation Package Sigma: Underlying Principles and a Rhombus Tiling Application
}

\author{
Carsten Schneider $]^{\dagger}$ \\ Research Institute for Symbolic Computation \\ Johannes Kepler University Linz. \\ Altenberger Str. 69 \\ A-4040 Linz, Austria \\ Carsten.Schneidererisc.uni-linz.ac.at
}

received Aug 21, 2003, revised Apr 16, Aug 26, 2004, accepted Aug 31, 2004.

\begin{abstract}
We give an overview of how a huge class of multisum identities can be proven and discovered with the summation package Sigma implemented in the computer algebra system Mathematica. General principles of symbolic summation are discussed.

We illustrate the usage of Sigma by showing how one can find and prove a multisum identity that arose in the enumeration of rhombus tilings of a symmetric hexagon. Whereas this identity has been derived alternatively with the help of highly involved transformations of special functions, our tools enable to find and prove this identity completely automatically with the computer.
\end{abstract}

Keywords: symbolic summation, rhombus tilings

\section{Introduction}

The overall object of this article is to give an introductory overview of how a huge class of multisum identities can be proven and discovered with the summation package Sigma [Sch01], which is based on the computer algebra system Mathematica. The algebraic platform of Sigma is built on the constructive difference field theory of $\Pi \Sigma$-fields [Kar81, Kar85, Bro00, Sch00, Sch01, Sch04b] that not only allows to simplify indefinite and definite sums of ( $q$-)hypergeometric terms, like [Gos78, Zei90, PS95a, PWZ96, PR97], but of $\Pi \Sigma$-terms, i.e., rational terms of arbitrarily nested indefinite sums and products. Due to the generality of $\Pi \Sigma$-terms, this opens up a new class of symbolic summation problems that cannot be treated by the algorithms and implementations [Weg97, Rie03] developed for ( $q$-)hypergeometric multisums or by those [CS98, Chy00] developed for holonomic and $\partial$-finite terms.

\footnotetext{
${ }^{\dagger}$ Supported by the Austrian Academy of Sciences, the SFB-grant F1305 of the Austrian FWF and by grant P16613-N12 of the Austrian FWF

1365-8050 (c) 2004 Discrete Mathematics and Theoretical Computer Science (DMTCS), Nancy, France
} 
In the first part of this article we shall discuss relevant techniques of symbolic summation, and we shall explain how these ideas can be applied in the difference field setting of $\Pi \Sigma$-fields and in difference ring extensions like $(-1)^{n}$; see [Sch01, Sch04b]. More precisely, this means that sequences, that may consist of rational terms of arbitrarily nested indefinite sums and products, are translated in a natural way into the corresponding difference field/ring setting [Kar85, Sch04b], and, by using a very general algebraic machinery [Kar81, AP94, Bro00, Sch02b, Sch04a, Sch02a, Sch04c], the corresponding summation principles (telescoping, creative telescoping, solving recurrences) are applied in this setting.

This allows to carry over Zeilberger's paradigm from hypergeometric terms [PWZ96] to so-called $\Pi \Sigma$ terms: given a definite nested multisum, find a recurrence and, if possible, solve the recurrence in terms of simpler expressions than the definite sum itself. Then the right combination of that solutions might give a closed form evaluation of the definite sum itself. The interplay of these summation techniques in the difference field setting can be summarized with the definite summation spiral that is graphically illustrated in Figure 1

In the second part of this article we shall demonstrate how these summation techniques within Sigma enable the user to find an alternative, completely automatic proof of a non-trivial multisum identity that arises in [FK00]. In this article, M. Fulmek and C. Krattenthaler count the number of rhombus tilings of a symmetric hexagon with side lengths $N, M, N, N, M, N$, with $N$ and $M$ having the same parity, which contain a particular rhombus next to the center of the hexagon. Within this counting there arises the subproblem of finding a closed form evaluation $S_{n}^{(1)}+\frac{n !}{(n+3) !} S_{n}^{(2)}$ for the two sums

$$
S_{n}^{(1)}:=\sum_{k=0}^{n-1} \frac{(-1)^{n+k}(n+k+4) ! \sum_{j=0}^{k} \frac{1}{j+1}}{(k+2) !(k+3) !(n-k-1) !}, S_{n}^{(2)}:=\sum_{k=0}^{n-1} \frac{(-1)^{k}(n+k+4) !\left(1-(-1)^{n}(n+2)\right)}{(k+1)(k+2) !^{2}(n-k-1) !} .
$$

In order to achieve this, the authors in [FK00] derive closed form evaluations for these sums, namely,

$$
S_{n}^{(1)}=-\frac{1+(-1)^{n}\left(n^{2}+3 n+3\right)}{n+2}-\left(1-(-1)^{n}(n+2)\right) \sum_{k=0}^{n} \frac{2}{k+1}
$$

and

$$
S_{n}^{(2)}=\left(1-(-1)^{n}(n+2)\right)\left(\sum_{k=0}^{n} \frac{2}{k+1}-\frac{(-1)^{n}+2 n^{3}+11 n^{2}+19 n+11}{(n+1)(n+2)(n+3)}\right),
$$

which finally delivers the identity

$$
S_{n}^{(1)}+\frac{n !}{(n+3) !} S_{n}^{(2)}=(-1)^{n}(n+2)-2
$$

for all $n \geq 0$. However, the proof of this identity, given in [FK00, Lemma 26], fills about four pages involving highly complicated transformations of special functions.

In [FK00], the authors were already aware that the sum identity (3) could be proven with a prototype version [Sch00] of our Sigma package [Sch01]. At that time, we were able to derive recurrence relations for the two sums $S_{n}^{(1)}$ and $S_{n}^{(2)}$. Afterwards, we combined those two recurrences using "gfun" (see [SZ94], or [Mal96] for a Mathematica implementation) to one recurrence of order 10 which contains 
$S_{n}^{(1)}+\frac{n !}{(n+3) !} S_{n}^{(2)}$ as a solution. It is then a simple task to check that the right hand side of identity (3) is also a solution of this combined recurrence. The fact that both sides of the equation (3) agree with the first 10 initial values finally shows the correctness of $(3)$. However, at that time we were not able to find the explicit evaluations (1) and (2). This has been changed partially in [Sch00], where we could find those evaluations by assuming that the right hand sides of (1) and (2) depend on the harmonic numbers $H_{n}=\sum_{i=1}^{n} \frac{1}{i}$.

In this article we shall show that meanwhile also the task of finding the evaluations (1) and (2) can be carried out with the summation package Sigma, without any guessing part, but only with computer algebra methods. In other words, we present an alternative proof of [FK00, Lemma 26] that not only shows the correctness of identity (3), but also delivers the explicit evaluations of the sums in (1) and 22. Moreover we shall illustrate that the proof of [FK00, Lemma 26] becomes completely automatic, if one uses Sigma, and hence feasible without advanced knowledge of hypergeometric functions and their transformations.

In principle, a reader may jump directly to the rhombus tiling application in Section 3 At every algorithmic step there, a pointer to the appropriate subsection of Section 2 is given where the ideas behind are outlined.

\section{Symbolic Summation in Difference Fields}

Symbolic summation usually is divided into two different subbranches, namely indefinite and definite summation. In contrast to indefinite summation, definite summation problems have closed form evaluations only for specifically chosen summation ranges. For instance, the sum $\sum_{k=a}^{b}\left(\begin{array}{l}n \\ k\end{array}\right)$ in general cannot be simplified further, whereas for the specific bounds $a=0$ and $b=n$ that sum evaluates to $2^{n}$.

In the following two subsections we will explain in more details, how indefinite and definite summation can be treated with the summation package Sigma [Sch01]. Moreover, additional information is given in " $\Pi \Sigma$-Remarks" how the summation problems are rephrased internally in the difference field setting of $\Pi \Sigma$-fields. Finally, we will summarize all these underlying difference field aspects in Subsection 2.3

\subsection{Indefinite summation}

Indefinite summation deals with the problem of eliminating summation quantifiers without using any knowledge about the summation range. More precisely, following [PS95b], we are interested in the following problem. Given an indefinite sum $\sum_{k=0}^{n} f(k)$ where $f$ belongs to some "nice" domain of sequences and $f(k)$ is independent of $n$. Find $g(k)$ in the same class or some suitable extension of it such that

$$
\sum_{k=0}^{n} f(k)=g(n)
$$

Alternatively, indefinite summation asks for solving

$$
\text { Problem T: Telescoping. }
$$

Given $f(k)$; find $g(k)$ such that

$$
g(k+1)-g(k)=f(k)
$$

holds within a certain range of $k$. 
Then, given such a telescoper $g(k)$ of $f(k)$, one derives by telescoping

$$
\sum_{k=a}^{b} f(k)=g(b+1)-g(a)
$$

if $b-a \in \mathbb{N}_{0}$.

There are various algorithms that solve Problem T for "nice" domains of sequences $f(k)$, like [Gos78, PS95a] for hypergeometric terms, [PR97] for $q$-hypergeometric terms, or [Chy00] for holonomic and $\partial$-finite terms.

In the summation package Sigma the sequences $f(k)$ and its telescoper $g(k)$ are described in the algebraic setting of difference fields, more precisely of $\Pi \Sigma$-fields [Kar81, Kar85], and certain difference rings; for more details see $\Pi \Sigma$-Remark 1 . This domain of sequences essentially covers $(q-)$ hypergeometric terms, see [Sch04b], and an important subclass of holonomic and $\partial$-finite terms that occurs frequently in symbolic summation. More generally, our approach allows to formulate sequences in terms of rational expressions consisting of arbitrarily nested indefinite sums and products that are out of scope of [Gos78, PR97, CS98, Chy00].

Without going into more details, we call all those sequences $f(k) \Pi \Sigma$-terms (in $k$ ) that can be described in terms of $\Pi \Sigma$-fields. Typical examples for $\Pi \Sigma$-terms are for instance

$$
\begin{array}{r}
H_{k}=\sum_{i=1}^{k} \frac{1}{i}, \quad H_{k}^{(2)}=\sum_{i=1}^{k} \frac{1}{i^{2}}, \quad k !=\prod_{i=1}^{k} i, \quad\left(\begin{array}{l}
n \\
k
\end{array}\right)=\prod_{i=1}^{k} \frac{n-i+1}{i}, \\
\left(1+H_{k}(n-2 k)\right)\left(\begin{array}{l}
n \\
k
\end{array}\right), \quad H_{k}^{2} H_{k}^{(2)}, \quad H_{k} \sum_{i=1}^{k} \frac{\prod_{j=1}^{i}\left(H_{j}^{2}+j !\right)}{H_{j}^{3}+j !} .
\end{array}
$$

One of the crucial properties of a $\Pi \Sigma$-term $f(k)$ is that the sums and products in the shifted version $f(k+1)$ can be expressed by the sums and products given in $f(k)$, like $H_{k+1}=H_{k}+\frac{1}{k+1}$ or $(k+1) !=(k+1) k !$. On the contrary, sums like $\sum_{i=1}^{k} \frac{H_{i}}{(i+k)^{4}}$ are not in the scope of $\Pi \Sigma$-terms.

$\Pi \Sigma$-Remark 1. In the sequel a brief introduction of $\Pi \Sigma$-fields is given; further information can be found in [Kar81, Kar85, Bro00, Sch01, Sch02b, Sch04b]. A difference field [Coh65], usually denoted by $(\mathbb{F}, \sigma)$, is nothing else than a field $\mathbb{F}$ together with a field automorphism $\sigma: \mathbb{F} \rightarrow \mathbb{F}$. Karr built up a difference field theory in a completely constructive manner that enables one to describe a huge class of nested multisums. In short, the class of $\Pi \Sigma$-fields contains difference fields $(\mathbb{F}, \sigma)$ that can be defined as follows. Basically $\mathbb{F}$ is constructed by a tower of finite field extensions $\mathbb{K}=\mathbb{E}_{0}<\mathbb{E}_{1}<\cdots<\mathbb{E}_{n}=\mathbb{F}$ with constant field $\mathbb{K}$, i.e., $\mathbb{K}=\left\{\sigma(g)=g \mid g \in \mathbb{F}_{i}\right\}$ for all $0 \leq i \leq n$. Moreover the following conditions for $1 \leq i \leq n$ hold: $\mathbb{E}_{i}:=\mathbb{E}_{i-1}\left(t_{i}\right)$ is a transcendental extension of $\mathbb{E}_{i-1}$ and we either have $\sigma\left(t_{i}\right)=a_{i} t_{i}$ (a product/ $\Pi$-extension) or $\sigma\left(t_{i}\right)=t_{i}+a_{i}$ (a sum extension) for some $a_{i} \in \mathbb{E}_{i-1} \backslash\{0\}$. In other words the class of $\Pi \Sigma$-fields contains difference fields such as $\mathbb{F}:=\mathbb{K}\left(t_{1}\right)\left(t_{2}\right) \ldots\left(t_{n}\right)$ where $\mathbb{F}$ is a field of rational functions over $\mathbb{K}$. Moreover these transcendental extensions allow to describe recursively defined nested sums and products in rational terms. Besides such product and sum extensions, a $\Pi \Sigma$-field can contain more general extensions of the type $\sigma\left(t_{i}\right)=\alpha_{i} t_{i}+\beta_{i}$ with $\alpha_{i}, \beta_{i} \in \mathbb{E} \backslash\{0\}$ together with some technical side conditions that are described further, for instance, in [Kar81, Kar85, Bro00, Sch01, Sch02b, Sch04b].

\# Throughout this article all fields will have characteristic 0 . 
Clearly, rational functions as $f(k) \in \mathbb{K}(k)$ with the shift operator $\sigma(k)=k+1$ are contained in the class of $\Pi \Sigma$-fields; also, most of the $\left(q\right.$-)hypergeometric terms like $f(k)=2^{k}$ or $f(k)=k$ ! can be rephrased in a $\Pi \Sigma$-field $(\mathbb{K}(k)(h), \sigma)$ with $\sigma(h)=2 h$ or $\sigma(h)=(k+1) h$; for more details see [Sch04b]. In particular, all the terms given in (6) can be formulated in $\Pi \Sigma$-fields.

On the other hand, frequently used objects like $(-1)^{k}$ cannot be formalized in $\Pi \Sigma$-fields, since we have the algebraic relation $\left((-1)^{k}\right)^{2}=1$. To overcome this problem, Sigma allows to handle objects like $\alpha^{k}, 1 \neq \alpha$ an $n$th root of unity, in ring extensions of the type $\mathbb{F}[x]$ where $(\mathbb{F}, \sigma)$ is a $\Pi \Sigma$-field with constant field $\mathbb{K}, \alpha \in \mathbb{K}, \sigma(x)=\alpha x$, and $x^{n}=1$. In particular, this means that $\sigma: \mathbb{F}[x] \rightarrow \mathbb{F}[x]$ is a ring automorphism, i.e., $(\mathbb{F}[x], \sigma)$ forms a difference ring, or a difference ring extension of $(\mathbb{F}, \sigma)$. For more details see [Sch01, Sch04b].

For instance, with Sigma one can produce the right hand sides of the identities

$$
\begin{aligned}
\sum_{k=0}^{a}\left(1+(n-2 k) H_{k}\right)\left(\begin{array}{l}
n \\
k
\end{array}\right) & =(n-a) H_{a}\left(\begin{array}{l}
n \\
a
\end{array}\right)+1, a \geq 0, \\
\sum_{k=0}^{a}\left(1+2(n-2 k) H_{k}\right)\left(\begin{array}{l}
n \\
k
\end{array}\right)^{2} & =\frac{(n-a)^{2}}{n^{2}}\left(1+2 n H_{a}\right)\left(\begin{array}{l}
n \\
a
\end{array}\right)^{2}, a \geq 0 ;
\end{aligned}
$$

note that the special case $a=n$ of theses identities is treated in [PS03]; see also [DPSW04a, CD04, KR04].

We illustrate the usage of our package Sigma by discovering and proving identity (7). First we start a Mathematica session by loading the package

$\ln [1]:=<<$ Sigma $^{\star}$

Sigma - A summation package by Carsten Schneider (C) RISC-Linz

and defining the sum $S(a)=$ mySum on the left hand side of (7) as follows:

$\operatorname{In}[2]:=\operatorname{mySum}=\operatorname{SigmaSum}[(\mathbf{1}+(\mathbf{n}-\mathbf{2 k}) \operatorname{SigmaHNumber}[\mathbf{k}]) \operatorname{SigmaBinomial}[\mathbf{n}, \mathbf{k}],\{\mathbf{k}, \mathbf{0}, \mathbf{a}\}]$

Out $[2]=\sum_{k=0}^{a}\left(1+(-2 k+n) H_{j}\right)\left(\begin{array}{l}n \\ k\end{array}\right)$.

Generally, the functions SigmaSum and SigmaProduct are used to define $\Pi \Sigma$-terms (in addition we allow summation objects like $(-1)^{n}$ that can be only formulated in difference ring extensions). For this purpose there are also several other functions available, like SigmaHNumber, SigmaBinomial or SigmaPower to define harmonic numbers, binomials or powers in terms of sums and products which itself can be converted into $\Pi \Sigma$-fields or certain difference ring extensions. For instance, SigmaHNumber [k] produces the $k$ th harmonic number $H_{k}$ which alternatively could be described by SigmaSum $[1 / i,\{i, 1, k\}]$.

Then, by applying the Sigma-function SigmaReduce to mySum $=S(a)$, we obtain the closed form evaluation:

$\ln [3]:=$ SigmaReduce $[\mathbf{m y S u m}]$

Out[3]= $1+(-\mathrm{a}+\mathrm{n}) \mathrm{H}_{\mathrm{a}}\left(\begin{array}{l}\mathrm{n} \\ \mathrm{a}\end{array}\right)$

$\Pi \Sigma$-Remark 2. Internally, the Sigma-package proceeds as follows. 1. Construction of the $\Pi \Sigma$-field $(\mathbb{F}, \sigma)$ : Take the rational function field $\mathbb{F}:=\mathbb{Q}(n)(k)(b)(h)$ and define the field automorphism $\sigma: \mathbb{F} \rightarrow \mathbb{F}$ 
by $\sigma(c)=c$ for $c \in \mathbb{Q}(n), \sigma(k)=k+1, \sigma(b)=\frac{n-k}{k+1} b$ and $\sigma(h)=h+\frac{1}{k+1}$. Note that the $k$-shifts $S_{k}\left(\begin{array}{l}n \\ k\end{array}\right)=$ $\left(\begin{array}{c}n \\ k+1\end{array}\right)=\frac{n-k}{k+1}\left(\begin{array}{l}n \\ k\end{array}\right)$ and $S_{k} H_{k}=H_{k+1}=H_{k}+\frac{1}{k+1}$ are reflected by the action of $\sigma$ on $b$ and $h$.

2. Solving the telescoping problem in $(\mathbb{F}, \sigma)$ : Sigma [Sch02b] finds the solution $g^{\prime}=b(h k-1)$ for the telescoping equation

$$
\sigma\left(g^{\prime}\right)-g^{\prime}=f^{\prime}
$$

with $f^{\prime}=b(1+(n-2 k) h)$. This means that $g(k)=\left(k H_{k}-1\right)\left(\begin{array}{l}n \\ k\end{array}\right)$ is a telescoper for $f(k)=(1+(n-$ $\left.2 k) H_{k}\right)\left(\begin{array}{l}n \\ k\end{array}\right)$.

Hence Sigma finds the telescoper $g(k)=\left(k H_{k}-1\right)\left(\begin{array}{l}n \\ k\end{array}\right)$ and the shifted version $g(k+1)=(n-k) H_{k}\left(\begin{array}{l}n \\ k\end{array}\right)$. The correctness of (4) for $0 \leq k \leq a$ is immediate and therefore the closed form is verified.

Similarly, one obtains a closed form of the sum

$\ln [4]:=\mathbf{m y S u m}=\sum_{\mathbf{k}=\mathbf{0}}^{\mathbf{n}}(\mathbf{3}+\mathbf{2} \mathbf{k})(-\mathbf{1})^{\mathbf{k}} \cdot \sum_{\mathbf{j}=\mathbf{1}}^{\mathbf{k}} \frac{\mathbf{1}+\mathbf{j}}{\mathbf{j}(\mathbf{2}+\mathbf{j})} ;$

by applying it to the function call SigmaReduce:

$\ln [5]:=$ SigmaReduce $[$ mySum]

Out[5] $=\frac{-3(1+n)(2+n)+2\left(3+3 n+n^{2}\right)(-1)^{n}+4(1+n)(2+n)^{2}(-1)^{n} \cdot \sum_{l_{1}=1}^{n} \frac{1+l_{1}}{l_{1}\left(2+l_{1}\right)}}{4(1+n)(2+n)}$

If one takes the shifted telescoper $g(k+1)$ of $f(k)=(3+2 k)(-1)^{k} \sum_{j=1}^{k} \frac{1+j}{j(2+j)}$ to be the expression in Out [5] with $n$ replaced by $k$, the proof of identity $\$$

$$
\sum_{k=0}^{n}(3+2 k)(-1)^{k} \sum_{j=1}^{k} \frac{1+j}{j(2+j)}=-\frac{3}{4}+\frac{\left(n^{2}+3 n+3\right)(-1)^{n}}{2(n+1)(n+2)}+(n+2)(-1)^{n} \sum_{i=1}^{n} \frac{i+1}{i(i+2)}
$$

for $n \geq 0$ can be carried out similarly to the proof of identity (7) from above.

In general, suppose that we are given a sum $S(a, b)=\sum_{k=a}^{b} f(k)$ with a $\Pi \Sigma$-term $f(k)$. If mySum $=$ $S(a, b)$ is defined with Sigma-functions as carried out in In $[2]$, by typing in

$$
\text { SigmaReduce[mySum] }
$$

one looks for a telescoper $g(k)$ in terms of sums and products that are given by the $\Pi \Sigma$-term $f(k)$. More precisely, first a $\Pi \Sigma$-field is constructed in which the sums and products occurring in $f(k)$ can be expressed formally. Afterwards one tries to solve the telescoping equation in this $\Pi \Sigma$-field. If such a $g(k)$ can be computed, by telescoping, see (5), the outermost summation quantifier in the sum $S(a, b)$ can be eliminated.

$\Pi \Sigma$-Remark 3. More precisely, the following difference field machinery is activated in Sigma; see also $\Pi \Sigma$-Remark 2. First a concrete $\Pi \Sigma$-field $(\mathbb{F}, \sigma)$ is constructed for the $\Pi \Sigma$-term $f(k)$ in (4). In particular, this means, one has to define a map which links the given summation objects, i.e., sequences $f(k)$, with elements $f^{\prime}$, say, in the constructed $\Pi \Sigma$-field; in other words, $f^{\prime} \in \mathbb{F}$ represents $f(k)$; for more details

$\S$ Note that this sum simplification will play an important role in Section 3 
see [Sch01, Chapter 2.5]. Given this translation machinery, it is decided constructively, if there exists a solution $g^{\prime} \in \mathbb{F}$ for the telescoping problem

$$
\sigma\left(g^{\prime}\right)-g^{\prime}=f^{\prime}
$$

If one finds such a $g^{\prime}$, one constructs a sequence $g(k)$ in terms of sums and products for which (4) holds. This finally gives the evaluation in (5).

Based on Karr's difference field theory [Kar81], the translation between $\Pi \Sigma$-terms and corresponding $\Pi \Sigma$ fields can be carried out completely automatically for most instances. Problematic cases can be treated by building up the underlying $\Pi \Sigma$-field manually; for more details see [Sch04b]. This user controlled construction can be achieved by calling SigmaReduce with the option Tower $\rightarrow\left\{\mathbf{s}_{1}(\mathrm{k}), \ldots, \mathbf{s}_{\mathrm{k}}(\mathrm{k})\right\}$, where $s_{i}(k)$ are $\Pi \Sigma$-terms in $k$. This means that Sigma first tries to construct the $\Pi \Sigma$-field for the term $s_{1}(k)$ and then extends the field in order to represent the remaining $s_{i}(k)$ following the input order; finally, the $\Pi \Sigma$-field is enlarged with necessary extensions in order to represent also $f(k)$.

Note that Sigma can also treat indefinite summation problems in terms of $(-1)^{k}$ that can be only treated in difference rings. For more details we refer to Subsection 2.3 .

We want to point out that so far we only dealt with indefinite summation problems where the telescoper $g(k)$ is searched in the domain given by the input sequence $f(k)$. But already for the slightly more general sum expression

$\ln [6]:=\mathbf{m y S u m}=\sum_{\mathbf{k}=\mathbf{0}}^{\mathbf{n}}(\mathbf{3}+\mathbf{2} \mathbf{k})(\mathbf{x})^{\mathbf{k}} \cdot \sum_{\mathbf{j}=\mathbf{1}}^{\mathbf{k}} \frac{\mathbf{1}+\mathbf{j}}{\mathbf{j}(\mathbf{2}+\mathbf{j})} ;$

we would fail to find such a telescoper in the ground field. Such kind of problems motivated us to generalize the indefinite summation approach to the following refined version [Sch04c] : with the Sigmapackage one is able to decide constructively if certain classes of sum extensions provide simpler solutions. More precisely, given a $\Pi \Sigma$-term $f(k)$, Sigma can search for a telescoper $g(k)$ of $f(k)$ that not only consists of sums and products given by $f(k)$ but that can contain sum extensions with the following property: they are not more nested than the given $\Pi \Sigma$-term $f(k)$ and their summands are composed by $\Pi \Sigma$-terms that occur in $f(k)$.

By setting the additional option SimplifyByExt $\rightarrow$ Depth in the function call SigmaReduce this refined algorithm can be activated.

$\ln [7]:=$ res $=$ SigmaReduce $[$ mySum, SimplifyByExt $\rightarrow$ Depth]

Out[7] $=\frac{x(-5-2 n+3 x+2 n x) x^{n} \cdot \sum_{l_{1}=1}^{n} \frac{1+l_{1}}{l_{1}\left(2+l_{1}\right)}-\sum_{l_{1}=1}^{n} \frac{\left(1+l_{1}\right)\left(-3+x-2 l_{1}+2 x l_{1}\right) x^{l_{1}}}{l_{1}\left(2+l_{1}\right)}}{(-1+x)^{2}}$

In this example Sigma finds the additional sum extension $E_{x}(n):=\sum_{k=1}^{n} \frac{(1+k)(-3+x-2 k+2 x k) x^{k}}{k(2+k)}$ that allows to find the closed form evaluation given in Out [7] with the same nested depth than the summand itself. (If one considers the special case $x=-1$, the sum $E_{-1}(x)$ can be simplified further to $3-\frac{2\left(3+3 n+n^{2}\right)(-1)^{n}}{(n+1)(n+2)}$ which finally gives 9 .)

II Analogously, this translation process can be controlled in the Sigma-functions CreativeTelescoping, GenerateRecurrence and SolveRecurrence that are explained later. 
$\Pi \Sigma$-Remark 4. In the difference field setting the following problem is solved in Sigma. First a $\Pi \Sigma$ field $(\mathbb{F}, \sigma)$ is constructed in which the $\Pi \Sigma$-term $f(k)$ can be represented with $f^{\prime} \in \mathbb{F}$. Then it is decided constructively, if there exists a bigger $\Pi \Sigma$-field $\left(\mathbb{F}\left(x_{1}, \ldots, x_{e}\right), \sigma\right)$ with $\sigma\left(x_{i}\right)-x_{i} \in \mathbb{F}$ and a $g^{\prime} \in \mathbb{F}\left(x_{1}, \ldots, x_{e}\right)$ with $\sigma\left(g^{\prime}\right)-g^{\prime}=f^{\prime}$ where $g^{\prime}$ is not more nested than $f^{\prime}$ itself. If Sigma finds such a $g^{\prime}$, it constructs a telescoper $g(k)$ of $f(k)$ in terms of additional sums whose depth is not larger than the $\Pi \Sigma$-term $f(k)$ itself. For algorithmic details we refer to [Sch01, Sch04c].

Further examples, like

$\ln [8]:=\mathbf{m y S u m}=\sum_{\mathbf{k}=\mathbf{0}}^{\mathbf{n}} \mathbf{H}_{\mathbf{k}}^{2} \mathbf{H}_{\mathbf{k}}^{(2)}$

$\ln [9]:=$ SigmaReduce[mySum, SimplifyByExt $\rightarrow$ Depth]

Out $[9]=\frac{1}{3}\left(-6 H_{n}+3 H_{n}^{2}-H_{n}^{3}+\left(3(1+2 n)-3(1+2 n) H_{n}+3(1+n) H_{n}^{2}\right) H_{n}^{(2)}+\sum_{i_{1}=1}^{n} \frac{1}{i_{1}^{3}}\right)$

and

$\ln [10]:=\operatorname{mySum}=\sum_{\mathbf{k}=\mathbf{1}}^{\mathbf{m}} \mathbf{H}_{\mathbf{k}} \sum_{\mathbf{j}=\mathbf{1}}^{\mathbf{k}} \frac{\prod_{\mathbf{i}=\mathbf{1}}^{\mathbf{j}}\left(\mathbf{H}_{\mathbf{i}}^{\mathbf{2}}+\mathbf{i} !\right)}{\mathbf{H}_{\mathbf{j}}^{\mathbf{3}}+(\mathbf{j} !)^{\mathbf{2}}} ;$

$\operatorname{In}[11]:=$ SigmaReduce[mySum, SimplifyByExt $\rightarrow$ Depth]

Out[11] $=\left(-m+(1+m) H_{m}\right) \sum_{l_{1}=1}^{m} \frac{\prod_{j=1}^{l_{1}}\left(H_{j}^{2}+j ! .\right)}{H_{l_{1}}^{3}+\mathfrak{l}_{1} !^{2}}-\sum_{\mathfrak{l}_{1}=1}^{m} \frac{\left(-\mathfrak{l}_{1}+H_{l_{1}} \mathrm{l}_{1}\right) \prod_{j=1}^{\mathrm{l}_{1}}\left(\mathrm{H}_{j}^{2}+j !\right)}{\mathrm{H}_{\mathrm{l}_{1}}^{3}+\mathrm{l}_{1} !^{2}{ }^{2}}$

show that these new ideas significantly enhance the algorithmic tool box.

\subsection{Definite summation and the definite summation spiral}

In general, the problem of definite summation is harder than indefinite summation, since in addition one also has to take into account the summation range. Up to now, all definite summation algorithms deal with such kind of problems by following Zeilberger's paradigm [PWZ96]: given a definite sum, find a recurrence (with polynomial coefficients) that contains the definite sum as a solution. If one can guess a closed form evaluation for a given definite sum, one may prove this identity by showing that the conjectured right hand side is also a solution of the computed recurrence and checking that the first initial values are the same.

More generally, one also tries to find solutions of a derived recurrence. Here the crucial point is that the computed solutions should be of a "simpler type" than the given definite sum expression. If one succeeds in this, one cannot only prove identities but even derive "closed form" evaluations.

Subsequently we will work out the interplay between those subproblems and methods that can be summarized with our definite summation spiral in Figure 1. Finally, a concrete example will illustrate these aspects in Section 3 . 


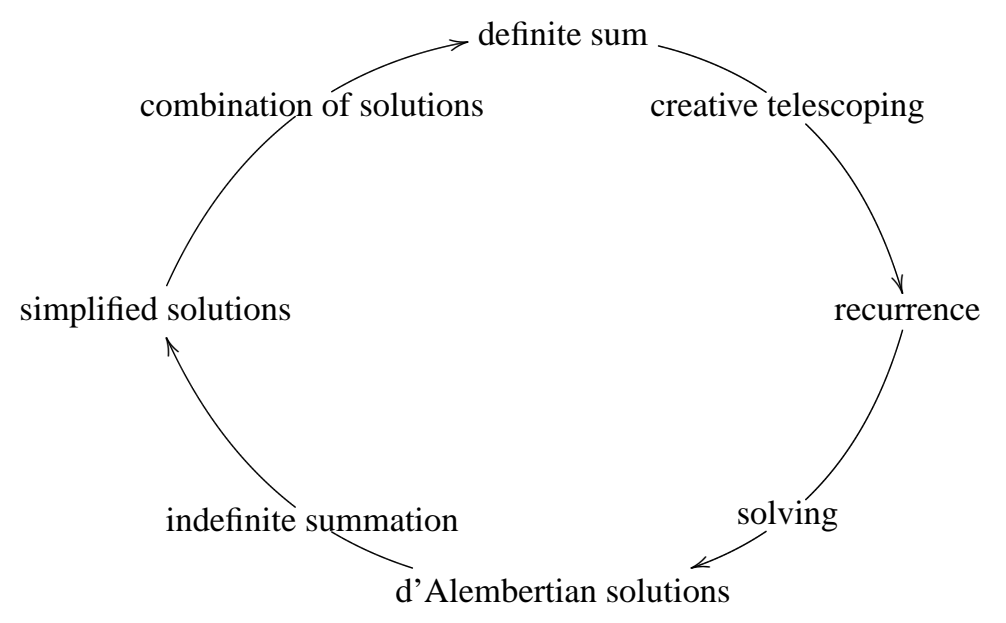

Fig. 1: The definite summation spiral.

\subsubsection{Creative telescoping}

The first step in our definite summation spiral consists of solving the following problem. Given a definite sum

$$
S(n):=\sum_{k=a}^{b} f(n, k)
$$

where $a, b$ are of the form $a=a_{1} n+a_{2}$ and $b=b_{1} n+b_{2}$ with $a_{1}, b_{1} \in \mathbb{Z}$ and $a_{2}, b_{2}$ independent of $n$. Find a recurrence of the form

$$
c_{0}(n) S(n)+\cdots+c_{d}(n) S(n+d)=h(n) .
$$

Most relevant summation algorithms accomplish this task by solving Problem CT or variations of it.

\section{Problem CT: Creative Telescoping.}

Given $f(n, k)$ and $d \in \mathbb{N}$; find $c_{0}(n), \ldots, c_{d}(n)$, free of $k$ and not all zero, and $g(n, k)$ such that

$$
g(n, k+1)-g(n, k)=c_{0}(n) f(n, k)+\cdots+c_{d}(n) f(n+d, k)
$$

holds within a certain range of $n$ and $k$.

The basic idea behind this is as follows. Suppose one succeeds in computing such $c_{i}(n)$ and $g(n, k)$ for given $f(n, k)$ and $d$. Then summing equation (13) over $k$ from $a$ to $b$ gives

$$
g(n, b+1)-g(n, a)=c_{0}(n) \sum_{k=a}^{b} f(n, k)+\cdots+c_{d}(n) \sum_{k=a}^{b} f(n+d, k) .
$$


Then with some mild extra conditions, one can express the sums $\sum_{k=a}^{b} f(n+i, k)$ in 13 in terms of $S(n+i)$. This implies a not necessarily homogeneous recurrence (12) for the definite sum $S(n)$. A concrete example in Remark2 2 illustrates in details how this transformation from (13) to (12) can be carried out.

Summarizing, solving Problem CT for a sequence $f(n, k)$ with a fixed $d \in \mathbb{N}$ enables one to construct a recurrence of order $d$ that contains the above defined sum $S(n)$ as solution. Note that $d$ must be specifically chosen for each attempt to solve Problem CT. Usually, one first tries to solve Problem CT for $d=1$, and increments $d$ until one finds a solution.

Originally, creative telescoping has been introduced in [Zei90] for hypergeometric terms $f(n, k)$ and $g(n, k)$; for a Mathematica implementation see for instance [PS95a]. Various other approaches in more general settings, like [PR97] for $q$-hypergeometric terms, [CS98, Chy00] for holonomic and $\partial$-finite terms, or [Weg97, Rie03] for ( $q$-)hypergeometric multisum terms follow this idea of creative telescoping or related paradigms.

With the summation package Sigma one can try to solve Problem CT for a given $d \in \mathbb{N}$ and a $\Pi \Sigma$ term $f(n, k)$ in $k$, which also depends on an extra parameter $n$, if the following property hold $\{$ also the shifted versions $f(n+i, k)$ for $1 \leq i \leq d$ are $\Pi \Sigma$-terms in $k$ and all those $\Pi \Sigma$-terms can be represented in a common $\Pi \Sigma$-field. Then, given such a $d$ and $f(n, k)$, one can search for a solution of Problem CT, where $g(n, k)$ consists of sums and products that occur in $f(n, k)$. Due to the generality of the input class of $\Pi \Sigma$-terms, this approach opens up the possibility to tackle various definite summation problems that cannot be treated by the earlier approaches [PR97, CS98, Chy00, Weg97, Rie03].

$\Pi \Sigma$-Remark 5. Given a $\Pi \Sigma$-term $f(n, k)$ and $d \in \mathbb{N}$, creative telescoping is handled in Sigma as follows. First a $\Pi \Sigma$-field $(\mathbb{F}, \sigma)$ is constructed with constant field $\mathbb{K}(n), n$ transcendental over $\mathbb{K}$, in which the $\Pi \Sigma$ terms $f(n+i, k)$ in $k$ can be expressed by $f_{i}^{\prime} \in \mathbb{F}$ for $0 \leq i \leq d$. Then one decides constructively, if there exist $c_{i}(n) \in \mathbb{K}(n)$, not all zero, and a $g^{\prime} \in \mathbb{F}$ with

$$
\sigma\left(g^{\prime}\right)-g^{\prime}=c_{0}(n) f_{0}^{\prime}+\cdots+c_{d}(n) f_{d}^{\prime}
$$

If one succeeds in finding such solutions $c_{i}(n)$ and $g^{\prime}$, a $\Pi \Sigma$-term $g(n, k)$ is constructed that gives a solution for Problem CT. We want to remark that with Sigma one can search for creative telescoping solutions also in algebraic difference ring extensions like $(-1)^{n}$.

Suppose that we are given $d \in \mathbb{N}$ and a definite sum $S(n)=\sum_{k=a}^{b} f(n, k)$ as in (11) where $f(n+i, k)$ is a $\Pi \Sigma$-term in $k$ for $0 \leq i \leq d$. Then, if mySum $=S(n)$ is defined with Sigma-functions as carried out in In [2], by typing in

$$
\text { creaSol }=\text { CreativeTelescoping }[\text { mySum, }, \text { RecOrder } \rightarrow \mathrm{d}]
$$

a set of creative telescoping solutions with $(13)$ is searched where each found solution is encoded in the form ${ }^{* *}\left\{\mathrm{c}_{0}(\mathrm{n}), \mathrm{c}_{1}(\mathrm{n}), \ldots, \mathrm{c}_{\mathrm{d}}(\mathrm{n}), \mathrm{g}(\mathrm{n}, \mathrm{k})\right\}$. Moreover, by entering

$$
\text { TransformToRecurrence[creaSol, mySum, n] }
$$

\footnotetext{
$\|$ Note that this property holds for almost all $\Pi \Sigma$-terms $f(n, k)$ in $k$.

** In our implementation the trivial solution $\{0, \ldots, 0,1\}$ with $1-1=0 f(n, k)+\cdots+0 f(n+d, k)$ is always included in the set of output solutions. There might be several non-trivial solutions, if $d$ is chosen too big.
} 
one obtains the resulting recurrences of the form (12) for the sum $S(n)$ that one can compute from the creative telescoping solutions. All these steps can be carried out in one stroke by using the Sigmafunction cal

$$
\text { GenerateRecurrence [mySum, } \mathrm{n}, \operatorname{RecOrder} \rightarrow \mathrm{d}] .
$$

As example we refer to the computation steps In [13], In [14] and In [22] in the Mathematica session that will be carried out in Section 3. Further examples can be found in [Sch01, PS03, DPSW04a, DPSW04b].

We want to emphasize that for our input class of $\Pi \Sigma$-terms, i.e., indefinite nested sums and products, we can verify the correctness of the obtained recurrence by the following recipe: check that the computed telescoping equation of Problem CT is correct for all $k$ with $a \leq k \leq b$. Then it suffices to verify that the inhomogeneous part $h(n)$ in $(12)$ is correctly determined. In Remark 2 we will illustrate with a concrete example how these verification steps can be carried out with the computer.

\subsubsection{Solving recurrences}

Suppose that we have derived a recurrence for a definite sum, say $S(k)$, of the type

$$
a_{m}(k) S(k+m)+\cdots+a_{0}(k) S(k)=b(k)
$$

where the coefficients $a_{i}(k)$ and the inhomogeneous part $b(k)$ are $\Pi \Sigma$-terms; note that exactly this type of recurrences can be computed with the Sigma-function call GenerateRecurrence. The next step in Figure 1 asks for solving the recurrence in terms of simpler expressions than the definite sum itself. Then the right linear combination of those solutions might give the closed form evaluation of the definite sum itself.

With the package Sigma there are various possibilities to achieve this task. The simplest strategy is to search for the solutions in the ground field given by the coefficients and the inhomogeneous part in (16). Namely, if a recurrence of the form (16) is inserted properly in the computer algebra system Mathematica, say in the variable rec like in In $[15$, , using the function call

$$
\text { SolveRecurrence }[\mathrm{rec}, \mathrm{S}[\mathrm{k}]]
$$

the user can look for all solutions in terms of sums and products given by the $a_{i}(k)$ and $b(k)$. The result of this function call is of the form

$$
\left\{\left\{0, \mathrm{~h}_{1}(\mathrm{k})\right\}, \ldots,\left\{0, \mathrm{~h}_{\mathrm{r}}(\mathrm{k})\right\}\right\} \text { or }\left\{\left\{0, \mathrm{~h}_{1}(\mathrm{k})\right\}, \ldots,\left\{0, \mathrm{~h}_{\mathrm{r}}(\mathrm{k})\right\},\{1, \mathrm{~g}(\mathrm{k})\}\right\}
$$

where $\left\{h_{1}(k), \ldots, h_{r}(k)\right\}$ gives a solution set of the homogeneous version of the recurrence and $g(k)$ gives a particular solution of the recurrence itself. Concrete applications can be found in the computation steps In [16] and In [24].

$\Pi \Sigma$-Remark 6. Internally, a $\Pi \Sigma$-field $(\mathbb{F}, \sigma)$ is constructed in which the coefficients $a_{i}(k)$ and the inhomogeneous part $b(k)$ can be expressed by $a_{i}^{\prime} \in \mathbb{F}$ and $b^{\prime} \in \mathbb{F}$. Then in Sigma all solutions $g^{\prime} \in \mathbb{F}$ with

$$
a_{m}^{\prime} \sigma^{m}\left(g^{\prime}\right)+\cdots+a_{0}^{\prime} g^{\prime}=b^{\prime}
$$

$\dagger$ If the option RecOrder $\rightarrow \mathrm{d}$ is omitted in the function calls CreativeTelescoping or GenerateRecurrence, Sigma tries to solve Problem CT first for $d=1$ and then for $d=2,3, \ldots$ until a solution is found; the termination is not guaranteed in this case. 
are searched. More precisely, a solution set $\left\{h_{1}^{\prime}, \ldots, h_{r}^{\prime}\right\} \subseteq \mathbb{F}$, linearly independent over the constant field $\{c \in \mathbb{F} \mid \sigma(c)=c\}$, is computed for the homogeneous version of (18). Moreover, a particular solution $g^{\prime} \in \mathbb{F}$ for $(18)$ is searched. The found solutions are then reinterpreted in form of $\Pi \Sigma$-terms $h_{i}(k), g(k)$ that give the solutions (17) for the original recurrence. Note that the search of the solutions for (18) can be also carried out in algebraic extensions like $(-1)^{k}$, i.e., the $a_{i}(k)$ and $b(k)$ may depend on $(-1)^{k}$.

In many instances the underlying difference field is too small in which the solutions $S(k)$ are searched. Therefore, Sigma provides the possibility to extend the underlying solution domain manually. Namely, by the function call

$$
\text { SolveRecurrence }\left[\mathrm{rec}, \mathrm{S}[\mathrm{k}] \text {, Tower } \rightarrow\left\{\mathrm{s}_{1}, \ldots, \mathrm{s}_{\mathrm{e}}\right\}\right]
$$

one can search for all solutions $S(k)$ in terms of sums and products occurring in the $a_{i}(k)$ and $b(k)$ together with the additional sums and products given by $s_{i}(k)$; see also $\Pi \Sigma$-Remark 3 The application of this feature is demonstrated in the computation steps In [19], In [25], and In [28] .

However, the guessing of additional $\Pi \Sigma$-terms is a highly non-trivial task. In order to dispense the user from extending the underlying difference field manually, the following two possibilities should be applied.

- Finding (q-)hypergeometric solutions. Due to the pioneering work [Pet92, vH98, APP98], one has powerful solvers in hand that allow to find all solutions $S(k)$ in $(q-)$ hypergeometric terms of a homogeneous recurrence with polynomial coefficients in $k$ or $q^{k}$. These solvers perfectly complement the summation package Sigma.

- Finding nested sum solutions and d'Alembertian solutions. With the function call

$$
\text { SolveRecurrence }\left[\mathrm{rec}, \mathrm{S}[\mathrm{k}] \text {, Tower } \rightarrow\left\{\mathrm{s}_{1}, \ldots, \mathrm{s}_{\mathrm{e}}\right\} \text {, NestedSumExt } \rightarrow \infty\right]
$$

the user can compute all nested sum solutions of a given recurrence rec of the form

$$
\sum_{k_{1}=0}^{n} b_{1}\left(k_{1}\right) \sum_{k_{2}=0}^{k_{2}} b_{2}\left(k_{2}\right) \cdots \sum_{k_{r}=0}^{k_{r-1}} b_{r}\left(k_{r}\right)
$$

where the $b_{i}\left(k_{i}\right)$ are $\Pi \Sigma$-terms in terms of sums and products given by the $s_{i}$ and by the $a_{i}(k)$ and $b(k)$ in the recurrence (16). Typical sum solutions can be found in out [17] and out [26] .

Remark 1. Internally, those solutions can be obtained by factorizing its linear difference equation as much as possible into linear right factors over the given difference field or ring; then each factor corresponds basically to one indefinite summation quantifier; see [AP94, Sch01]. An important result is that the class of "linearly" nested sum solutions (19) over the given $\Pi \Sigma$-terms contains also all solutions that consist of rational terms of arbitrarily nested sums over the given $\Pi \Sigma$-terms; for the rational case see [HS99] and for the general $\Pi \Sigma$-field case see [Sch01]. Note that the class of sum solutions is contained in the class of d'Alembertian solution [AP94] which again is included in the class of Liouvillian solutions [HS99].

An important special case is the "rational case", i.e., the coefficients of the recurrence are in the field $\mathbb{K}(k)$ with the shift operator $S(k)=k+1$. Then the d'Alembertian solutions are of the type (19) where $b_{i}\left(k_{i}\right)$ are hypergeometric terms over $\mathbb{K}\left(k_{i}\right)$. Here the crucial observation is that a hypergeometric term solution of a recurrence gives also a linear right factor of a recurrence. Therefore, the application of algorithms like [Pet92, vH98] might contribute to a refined factorization of a given recurrence into linear right factors, and thus to further solutions of the recurrence; see [AP94, Sch01]. In combination with [AP94, Sch01] 
and manual extensions of the solution domain (with the option Tower), the user can compute all those d'Alembertian solutions with the summation package Sigma; for further details and illustrative examples we refer to [PS03, DPSW04b].

\subsubsection{Indefinite summation}

Nested sum solutions and d'Alembertian solutions consist of non-trivial and highly nested indefinite sums of the form (19). If such solutions contribute to the closed form evaluation of the original definite sum expression, in most instances the found evaluation is not simpler, but even more complex, namely more nested. In order to overcome this problem, one has to reduce those nested sums to expressions which are less nested than the originally given definite multisum. It turns out that all nested sum solutions and many d'Alembertian solutions can be expressed in $\Pi \Sigma$-fields or difference ring extensions like $(-1)^{k}$; see [Sch04b]. In this case one can apply our indefinite summation algorithms described in Section 2.1 in order to simplify those sum solutions and d'Alembertian solutions further. This simplification step is carried out, for instance, in In [18] and In [27].

\subsubsection{Combination of solutions.}

Now assume that we managed to compute a recurrence of order $d$ for a definite sum $S(n)$ that holds for all $n \geq n_{0}, n_{0}$ an integer, and we found a set of solutions of that recurrence that holds for all $n \geq n_{0}$. More precisely, suppose that in a Mathematica session mySum stands for our definite sum $S(n)$ and recSol for our set of solutions of the recurrence that is given in the form (17) with $k$ replaced by $n$. Then with

$$
\text { FindLinearCombination[recSol, mySum, d, MinInitialValue } \left.\rightarrow \mathrm{n}_{0}\right]
$$

the user can try to find a linear combination of the solutions of the homogeneous version of the recurrence plus one particular solution of the inhomogeneous recurrence that evaluates to the same initial values for $n \in\left\{n_{0}, n_{0}+1, \ldots, n_{0}+d-1\right\}$ as the given definite sum. If Sigma succeeds in finding such a linear combination, this expression equals $S(n)$ for all $n \geq n_{0}$. Note that Sigma might fail to find this linear combination if a particular solution or some solutions of the homogeneous version of the recurrence are missing in recSol.

\subsection{The "Master Problem" for symbolic summation in difference fields}

The summation problems sketched in the previous $\Pi \Sigma$-Remarks can be summarized by

Problem PLDE: Solving Parameterized Linear Difference Equations.

Given a $\Pi \Sigma$-field $(\mathbb{F}, \sigma)$ with constant field $\mathbb{K}, a_{0}, \ldots, a_{m} \in \mathbb{F}$, and $f_{0}, \ldots, f_{d} \in \mathbb{F}$;

find all $g \in \mathbb{F}$ and all $c_{0}, \ldots, c_{d} \in \mathbb{K}$ with $a_{m} \sigma^{m}(g)+\cdots+a_{0} g=c_{0} f_{0}+\cdots+c_{d} f_{d}$.

Namely, specializing to $d=0$ and $m=1$ with $a_{1}=1$ and $a_{2}=-1$, one considers the telescoping problem (10) for indefinite summation. Moreover, specializing to $m=1$ with $a_{1}=1$ and $a_{2}=-1$, one can formulate the creative telescoping problem $(15)$ if $\mathbb{K}=\mathbb{K}^{\prime}(n)$ and $f_{i} \in \mathbb{F}$ stands for the $\Pi \Sigma$-term $f(n+i, k) \in \mathbb{F}$ in $k$ for $0 \leq i \leq d$. Furthermore, if one sets $d=0$, one considers the problem to solve linear difference equations (18) of order $m$. 
In [Kar81, Kar85], M. Karr developed a complete algorithm that solves Problem T in the general $\Pi \Sigma$-field setting; only some additional properties are required for the constant field, that are worked out in [Sch04b]. In some sense, Karr's algorithm [Kar81] is the summation counterpart to Risch's algorithm [Ris69, Ris70] for indefinite integration.

In [Sch00, Sch01], it was observed for the first time that Karr's algorithm not only can solve Problem T but also Problem CT in $\Pi \Sigma$-fields. More precisely, Karr's algorithm can solve Problem PLDE with $m=1$. Analogously to the fact that the extended version of Gosper's algorithm [Zei90] represents a significant generalization to definite hypergeometric summation, with this observation Karr's algorithm can be viewed as a major step forward with respect to definite summation in general.

Based on results in [Bro00], Karr's algorithm was streamlined in [Sch01, Sch02b] to a more compact and efficient algorithm. Moreover, in [Sch02b, Sch04a, Sch02a] together with results from [Bro00], this streamlined algorithm was generalized to a method that enables the user to search for all solutions of Problem PLDE for an arbitrary order $m$. Although there are still open problems in the resulting algorithms, one finds eventually all the solutions for Problem PLDE by repeating the computation process and increasing step by step the range in which the solutions may exist; these ideas are presented in [Sch02b].

Furthermore we want to emphasize that Sigma provides methods that enable the user to search for solutions of Problem PLDE in difference ring extensions, like $(-1)^{k}$, that contain zero-divisors, like $(1-$ $\left.(-1)^{k}\right)\left(1+(-1)^{k}\right)=0$; for more details see [Sch01]. Those ideas are partially needed in the computation steps In [5], In [18], In [19], In [25], and In [28] .

\section{A Rhombus Tiling Application}

In the sequel we will prove the multisum identities (11) and (2) that arise in [FK00]. Following our definite summation spiral in Figure 1, those identities will not only be proven with our package Sigma, but we will also find their right hand sides.

First we set up the summation problem $S_{n}^{(1)}=$ mySum1 as carried out in In [2] .

$\operatorname{In}[12]:=\mathbf{m y S u m} 1=\sum_{\mathbf{k}=\mathbf{1}}^{\mathbf{n}}-\frac{\mathbf{H}_{\mathbf{k}}(\mathbf{3}+\mathbf{k}+\mathbf{n}) ! \cdot(-\mathbf{1})^{\mathbf{k}} \cdot(-\mathbf{1})^{\mathbf{n}}}{(\mathbf{1}+\mathbf{k}) !^{\mathbf{2}}(\mathbf{2}+\mathbf{k})(-\mathbf{k}+\mathbf{n}) !} ;$

\section{Finding a recurrence with creative telescoping}

Given this sum expression, we are able to compute a recurrence relation of order three by solving the creative telescoping problem; see Problem CT.

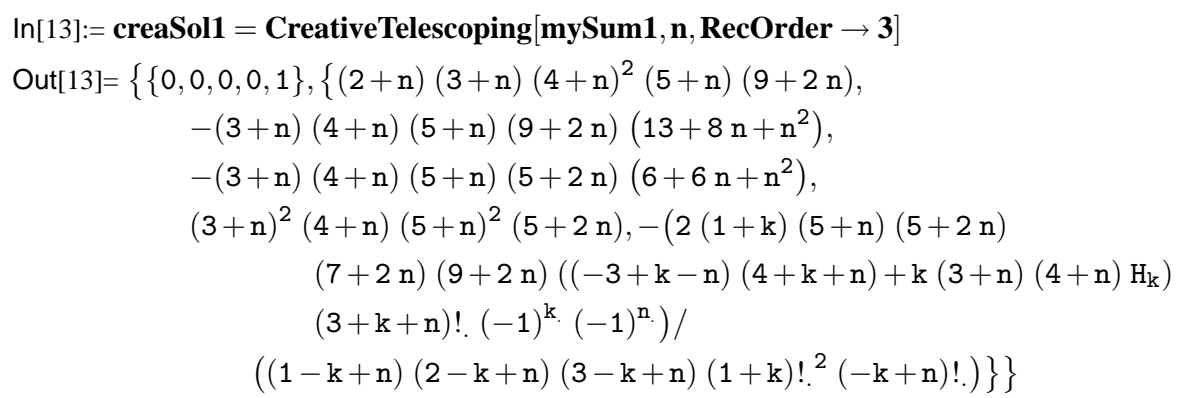


Here the second entry in the output of Out [13], say $\left\{c_{0}(n), c_{1}(n), c_{2}(n), c_{3}(n), g(n, k)\right\}$, gives the solution of Problem CT for $d=3$ and the summand

$$
f(n, k)=-\frac{H_{k}(n+k+3) !(-1)^{k}(-1)^{n}}{(k+2)(k+1) !^{2}(n-k) !}
$$

of $S_{n}^{(1)}=\sum_{k=1}^{n} f(n, k)$. Then, as described in Subsection 2.2.1, we can generate from this result a recurrence for $S_{n}^{(1)}$ with the function call

In[14]:= TransformToRecurrence $[$ creaSol1, mySum1, $\mathbf{n}$ ]

$$
\begin{aligned}
& \text { Out[14] }=\left\{(1+n)(2+n)^{2}(3+n)(4+n)(9+2 n) n ! . \operatorname{SUM}[n]-\right. \\
& (1+n)(2+n)(3+n)(9+2 n)\left(13+8 n+n^{2}\right) n ! \text { SUM }[1+n]- \\
& (1+n)(2+n)(3+n)(5+2 n)\left(6+6 n+n^{2}\right) n ! \operatorname{SUM}[2+n]+ \\
& (1+n)(2+n)(3+n)^{2}(5+n)(5+2 n) n ! S \operatorname{SUM}[3+n]== \\
& \left.-2(5+2 n)(7+2 n)(9+2 n)(3+n) !(-1)^{n}\right\}
\end{aligned}
$$

This means that $\operatorname{SUM}[\mathrm{n}]=S_{n}^{(1)}$ (=mySum1) satisfies the output recurrence Out [14]. We could also carry this out in one step by the call GenerateRecurrence[mySum, $n, \operatorname{RecOrder~} \rightarrow 3$ ] which just gives the same recurrence as in out [14].

We want to emphasize that the user can verify the correctness of recurrences independently of the steps of the algorithm, see the following remark.

Remark 2. With the $c_{i}(n)$ and $g(n, k)$ given in Out [13] one can show that $S_{n}^{(1)}$ is a solution of the recurrence out [14] as follows. For [20) observe that $f(n+i, k)=f(n, k) f_{i}$ where $f_{0}=1$,

$$
f_{1}=-\frac{n+4+k}{n+1-k}, \quad f_{2}=\frac{(n+4+k)(n+5+k)}{(n+1-k)(n+2-k)}, \quad f_{3}=-\frac{(n+4+k)(n+5+k)(n+6+k)}{(n+1-k)(n+2-k)(n+3-k)} .
$$

Moreover note that the $\Pi \Sigma$-term $g(n, k)$ shifted in $k$ can be rewritten as

$$
\begin{array}{r}
g(n, k+1)=-\frac{2(k+1)(n+5)(n+4+k)(2 n+5)(2 n+7)(2 n+9)}{(k+2)(n+2-k)} \\
\quad \times\left(H_{k}\left(n^{2}+7 n+12\right)+k+2\right) \frac{(n+k+3) !(-1)^{k}(-1)^{n}}{(k+1) !^{2}(n-k) !}
\end{array}
$$

by using the relations $H_{n+1}=H_{n}+\frac{1}{n+1}$ and $(-1)^{n+1}=-(-1)^{n}$. Then with these representations, we verify that 13 with $d=3$ holds for all $0 \leq k \leq n$. First we check that there do not occur any poles during the evaluation in the chosen representations of $g(n, k), g(n, k+1)$ and $f(n+i, k)$ for $0 \leq i \leq 3$ within the range $0 \leq k \leq n$. Then we substitute those specific terms in $g(n, k+1)-g(n, k)-\left(c_{0}(n) f(n, k)+\right.$ $\left.\cdots+c_{3}(n) f(n+3, k)\right)$, bring these expressions over a common denominator, and check symbolically that the polynomial expression in the numerator vanishes. This shows the correctness of (13) for $0 \leq k \leq n$. Moreover, summing equation (13) over $k$ from 0 to $n$ gives

$$
c_{0}(n) \sum_{k=0}^{n} f(n, k)+\cdots+c_{3}(n) \sum_{k=0}^{n} f(n+3, k)=g(n, n+1)-g(n, 0) .
$$


Then with

$$
S_{n+i}^{(1)}=\sum_{k=0}^{n} f(n+i, k)+\sum_{j=1}^{i} f(n+i, n+j)
$$

for $i \geq 0$, the correctness of the recurrence rec with SUM $[\mathrm{n}]=S_{n}^{(1)}$ follows for all $n \geq 0$.

Dividing the output recurrence in Out [14] by the non-zero factor $(n+3)(n+2)(n+1) n$ ! (for $n \geq 0$ ) gives the simplified version:

$$
\begin{aligned}
\operatorname{In}[15]:=\operatorname{rec1} & =(\mathbf{2}+\mathbf{n})(\mathbf{4}+\mathbf{n})(\mathbf{9}+\mathbf{2} \mathbf{n}) \operatorname{SUM}[\mathbf{n}]-(\mathbf{9}+\mathbf{2} \mathbf{n})\left(\mathbf{1 3}+\mathbf{8} \mathbf{n}+\mathbf{n}^{\mathbf{2}}\right) \operatorname{SUM}[\mathbf{1}+\mathbf{n}]- \\
& (\mathbf{5}+\mathbf{2} \mathbf{n})\left(\mathbf{6}+\mathbf{6} \mathbf{n}+\mathbf{n}^{\mathbf{2}}\right) \mathbf{S U M}[\mathbf{2}+\mathbf{n}]+(\mathbf{3}+\mathbf{n})(\mathbf{5}+\mathbf{n})(\mathbf{5}+\mathbf{2} \mathbf{n}) \operatorname{SUM}[\mathbf{3}+\mathbf{n}]= \\
& -\mathbf{2}(\mathbf{5}+\mathbf{2} \mathbf{n})(\mathbf{7}+\mathbf{2} \mathbf{n})(\mathbf{9}+\mathbf{2} \mathbf{n})(-\mathbf{1})^{\mathbf{n}} \cdot
\end{aligned}
$$

Solving the recurrence with sum solutions (d'Alembertian solutions)

In the next step we try to find solutions of the recurrence rec1 given in Out [15] . To accomplish this task, Sigma provides the following function call; see Subsection 2.2.2

$\operatorname{In}[16]:=$ SolveRecurrence $[\operatorname{rec1}, \mathbf{S U M}[\mathbf{n}]]$

Out $[16]=\left\{\{0,1\},\left\{0,(2+n)(-1)^{n} \cdot\right\}\right\}$

Internally Sigma constructs the underlying difference ring $\mathbb{A}=\mathbb{Q}(n)\left[(-1)^{n}\right]$ given by the objects in the recurrence and afterwards tries to solve the recurrence formulated in this algebraic setting $\mathbb{A}$. In this case Sigma finds two linearly independent solutions of the homogeneous version of the recurrence, namely 1 and $(n+2)(-1)^{n}$.

Obviously, those solutions are not sufficient to describe the whole set of solutions of the given recurrence. Therefore we try to extend the underlying difference ring in form of sum solutions by setting in addition the option NestedSumExt $\rightarrow \infty$; see Subsection 2.2.2.

$\operatorname{In}[17]:=$ SolveRecurrence $[$ rec1, SUM[n], NestedSumExt $\rightarrow \infty$, IndefiniteSummation $\rightarrow$ False]

$$
\begin{gathered}
\text { Out }[17]=\left\{\{0,1\},\left\{0,(2+n)(-1)^{n} \cdot\right\},\left\{0,-\sum_{l_{1}=0}^{n}\left(3+2 l_{1}\right)(-1)^{l_{1}} \cdot \sum_{l_{2}=1}^{l_{1}} \frac{(-1)^{l_{2}}}{l_{2}\left(2+l_{2}\right)}\right\},\right. \\
\left.\left\{1,2 \sum_{l_{1}=0}^{n}\left(3+2 l_{1}\right)(-1)^{l_{1}} \cdot \sum_{l_{2}=1}^{l_{1}} \frac{1+l_{2}}{l_{2}\left(2+l_{2}\right)}\right\}\right\}
\end{gathered}
$$

In this example Sigma succeeded completely since it was able to compute three linearly independent solutions of the homogeneous version of the recurrence and one particular solution of the inhomogeneous recurrence itself.

\section{Simplifying the solutions with indefinite summation}

Now the essential step is that those two sum solutions in Out [17] can be simplified further with Sigma's indefinite summation algorithm; see identity (9). By default, i.e., omitting the option Indef initeSummation $\rightarrow \mathrm{False}$, those sum solutions are simplified immediately which results in:

Remarks concerning the option Indef initeSummation $\rightarrow$ False are given in the next paragraph. 
$\ln [18]:=$ SolveRecurrence $[\operatorname{rec} 1, \operatorname{SUM}[\mathbf{n}]$, NestedSumExt $\rightarrow \infty]$

$$
\begin{aligned}
\text { Out }[18]=\{ & \{0,1\},\left\{0,(2+n)(-1)^{\mathrm{n}} \cdot\right\},\left\{0, \frac{-1-2(1+\mathrm{n}) \sum_{\mathrm{l}_{1}=1}^{\mathrm{n}} \frac{1+\mathrm{l}_{1}}{\mathrm{l}_{1}\left(2+\mathrm{l}_{1}\right)}}{2(1+\mathrm{n})}\right\}, \\
& \left\{1, \frac{\left.\left.\left(3+3 \mathrm{n}+\mathrm{n}^{2}\right)(-1)^{\mathrm{n} \cdot}+2(1+\mathrm{n})(2+\mathrm{n})^{2}(-1)^{\mathrm{n} \cdot \sum_{\mathrm{l}_{1}=1}^{\mathrm{n}} \frac{1+\mathrm{l}_{1}}{\mathrm{l}_{1}\left(2+\mathrm{l}_{1}\right)}}\right\}\right\}}{(1+\mathrm{n})(2+\mathrm{n})}\right.
\end{aligned}
$$

Looking closer at this result, from the partial fraction decomposition of the summand

$$
\sum_{i=1}^{n} \frac{i+1}{i(i+2)}=\frac{1}{2}\left(\sum_{i=1}^{n} \frac{1}{i}+\sum_{i=1}^{n} \frac{1}{i+2}\right)
$$

one sees immediately that this sum can be expressed in terms of the harmonic numbers $H_{n}$. This cosmetic change of the solution representation can be also achieved by solving the recurrence again in the solution domain extended with $H_{n}$.

$$
\begin{gathered}
\operatorname{In}[19]:=\text { recSol1 }=\text { SolveRecurrence }\left[\mathbf{r e c 1}, \mathbf{S U M}[\mathbf{n}], \text { Tower } \rightarrow\left\{\mathbf{H}_{\mathbf{n}}\right\}\right] \\
\text { Out }[19]=\left\{\{0,1\},\left\{0, \frac{3-\mathrm{n}^{2}+2(1+\mathrm{n})(2+\mathrm{n}) \mathrm{H}_{\mathrm{n}}}{(1+\mathrm{n})(2+\mathrm{n})}\right\},\left\{0,(2+\mathrm{n})(-1)^{\mathrm{n}} \cdot\right\},\right. \\
\left.\left\{1, \frac{\left(9+10 \mathrm{n}+3 \mathrm{n}^{2}+2(1+\mathrm{n})(2+\mathrm{n})^{2} \mathrm{H}_{\mathrm{n}}\right)(-1)^{\mathrm{n} .}}{(1+\mathrm{n})(2+\mathrm{n})}\right\}\right\}
\end{gathered}
$$

Remark 3. We want to point out that the correctness of the solutions in Out [ [19] for $n \geq 0$ (or of the representations Out [17] or Out [18] from above) can be verified similarly as in Remark 2 by substituting the solutions in the recurrence of out $[15]$ and checking equality for the resulting equation. For instance, for the solutions given in Out [19], this can be achieved by applying the relations $H_{n+1}=$ $H_{n}+\frac{1}{n+1}$ and $(-1)^{n+1}=-(-1)^{n}$.

\section{Finding a closed form evaluation by combining the solutions}

So far we computed a recurrence relation of order 3 for the definite $\operatorname{sum} S_{n}^{(1)}$, that holds for all $n \geq 0$ (see Remark 2), and found solutions for that recurrence, that hold for all $n \geq 0$ (see Remark 3). Therefore a closed form of $S_{n}^{(1)}$ can be obtained by composing the particular linear combination of the homogeneous solutions plus the inhomogeneous solution that matches the first three initial values of $S_{n}^{(1)}$ for $n=0,1,2$; see Subsection 2.2.4.

$\operatorname{In}[20]:=$ FindLinearCombination [recSol1, mySum1, 3, MinInitialValue $\rightarrow$ 0]

Out[20] $=\frac{-5-3 n-2(1+n)(2+n) H_{n}+\left(5+2 n-2 n^{2}-n^{3}+2(1+n)(2+n)^{2} H_{n}\right)(-1)^{n}}{(1+n)(2+n)}$

This shows that

$$
S_{n}^{(1)}=\frac{-5-3 n-2(1+n)(2+n) H_{n}+\left(5+2 n-2 n^{2}-n^{3}+2(1+n)(2+n)^{2} H_{n}\right)(-1)^{n}}{(1+n)(2+n)},
$$

or equivalently (1), holds for all $n \geq 0$. 
In the same spirit we are able to find a closed form evaluation for the hypergeometric sum

$$
T_{n}:=\sum_{k=0}^{n-1} \frac{(-1)^{k}(n+k+4) !}{(k+1)(k+2) !^{2}(n-k-1) !}
$$

where $S_{n}^{(2)}=\left(1-(-1)^{n}(n+2)\right) T_{n}$. More precisely, we first compute a recurrence for $T_{n}=$ mySum2.

$\ln [21]:=\mathbf{m y S u m} 2=\sum_{\mathbf{k}=\mathbf{1}}^{\mathbf{n}}-\frac{(\mathbf{3}+\mathbf{k}+\mathbf{n}) ! \cdot(-\mathbf{1})^{\mathbf{k}}}{\mathbf{k}(\mathbf{1}+\mathbf{k}) !^{\mathbf{2}}(-\mathbf{k}+\mathbf{n}) !} ;$

$\operatorname{In}[22]:=$ GenerateRecurrence [mySum $\mathbf{2}, \mathbf{n}$, RecOrder $\rightarrow \mathbf{2}$ ]

Out[22]= $\left\{(1+n)(3+n)(4+n)(7+2 n) n !\right.$ SUM[n] $+6(1+n)(3+n)^{2} n !$ SUM[1 $[1+n]-$

$$
(1+\mathrm{n})(2+\mathrm{n})(3+\mathrm{n})(5+2 \mathrm{n}) \mathrm{n} \text { ! SUM[2 }[2+\mathrm{n}]==-2(5+2 \mathrm{n})(7+2 \mathrm{n})(4+\mathrm{n}) !\}
$$

This means that $\operatorname{SUM}[\mathrm{n}]=T_{n}$ (=mySum2) satisfies the output recurrence Out [22] for $n \geq 0$. Given the creative telescoping solution, the verification of this recurrence relation is immediate and is omitted here. Note that this recurrence could have been computed with any other implementation that can deal with creative telescoping for definite hypergeometric sums [Zei90, PWZ96], like for instance [PS95a].

Dividing the output recurrence Out [22] by the non-zero term $(n+1)(n+3) n$ ! (for $n \geq 0)$ gives the simplified version:

$\ln [23]:=\operatorname{rec} 2=-(4+n)(7+2 \mathrm{n}) \operatorname{SUM}[\mathbf{n}]-\mathbf{6}(3+\mathbf{n}) \operatorname{SUM}[1+\mathbf{n}]+$

$$
(\mathbf{2}+\mathbf{n})(\mathbf{5}+\mathbf{2} \mathbf{n}) \mathbf{S U M}[\mathbf{2}+\mathbf{n}]==\mathbf{2}(\mathbf{2}+\mathbf{n})(\mathbf{4}+\mathbf{n})(\mathbf{5}+\mathbf{2} \mathbf{n})(\mathbf{7}+\mathbf{2} \mathbf{n}) ;
$$

Subsequently we solve the recurrence rec2 given in In [23.

- In the underlying algebraic setting of the recurrence we obtain the following solution

$\ln [24]:=$ SolveRecurrence[rec2, SUM[n]]

Out[24] $=\{\{0,(1+n)(2+n)(3+n)\}\}$

which gives just a solution of the homogeneous recurrence.

- Next, we ask for hypergeometric solutions of the homogeneous version of the recurrence. For instance, the implementations [Pet92, vH98] give the additional solution $(-1)^{n}$. This gives the following result.

$\operatorname{In}[25]:=$ SolveRecurrence $\left[\right.$ rec2, SUM $[\mathbf{n}]$, Tower $\left.\rightarrow\left\{(-\mathbf{1})^{\mathbf{n}}\right\}\right]$

Out $[25]=\left\{\{0,(1+\mathrm{n})(2+\mathrm{n})(3+\mathrm{n})\},\left\{0,(-1)^{\mathrm{n}} \cdot\right\}\right\}$

- Finally, we look for sum solutions of the recurrence and get additionally an inhomogeneous solution.

$\operatorname{In}[26]:=$ SolveRecurrence $\left[\right.$ rec2, $\operatorname{SUM}[\mathbf{n}]$, Tower $\rightarrow\left\{(-\mathbf{1})^{\mathbf{n}}\right\}$,

NestedSumExt $\rightarrow \infty$, IndefiniteSummation $\rightarrow$ False $]$

Out[26] $=\left\{\{0,(1+n)(2+n)(3+n)\},\left\{0,(-1)^{n} \cdot\right\}\right.$,

$$
\left.\left\{1,2(-1)^{\mathrm{n}} \cdot \sum_{\mathrm{l}_{1}=0}^{\mathrm{n}}\left(6+13 \mathrm{l}_{1}+9 \mathrm{l}_{1}^{2}+2 \mathrm{l}_{1}^{3}\right)(-1)^{\mathrm{l}_{1}} \cdot \sum_{\mathrm{l}_{2}=0}^{\mathrm{l}_{1}} \frac{1}{1+\mathrm{l}_{2}}\right\}\right\}
$$

Removing the option IndefiniteSummation $\rightarrow$ False in the previous function call, i.e., applying in addition Sigma's indefinite summation algorithm, leads to: 
$\operatorname{In}[27]:=$ SolveRecurrence $\left[\right.$ rec2, SUM $[\mathbf{n}]$, Tower $\rightarrow\left\{(-1)^{\mathbf{n}} \cdot\right\}$, NestedSumExt $\left.\rightarrow \infty\right]$

Out[27]= $\left\{\{0,(1+\mathrm{n})(2+\mathrm{n})(3+\mathrm{n})\},\left\{0,(-1)^{\mathrm{n}} \cdot\right\}\right.$,

$$
\left.\left\{1,1+3 n+n^{2}+2(1+n)(2+n)(3+n) \sum_{n_{1}=0}^{n} \frac{1}{1+l_{1}}\right\}\right\}
$$

- In the end, we just solve the recurrence again in terms of $H_{n}$ and $(-1)^{n}$ which gives:

$$
\begin{aligned}
\operatorname{In}[28]:= & \text { recSol2 }=\text { SolveRecurrence }\left[\text { rec2 }, \mathbf{S U M}[\mathbf{n}], \text { Tower } \rightarrow\left\{\mathbf{H}_{\mathbf{n}},(-\mathbf{1})^{\mathbf{n}} \cdot\right\}\right] \\
\text { Out }[28]=\left\{\{0,(1+\mathrm{n})(2+\mathrm{n})(3+\mathrm{n})\},\left\{0,(-1)^{\mathrm{n}} \cdot\right\},\right. & \\
& \left.\left\{1,13+13 \mathrm{n}+3 \mathrm{n}^{2}+2(1+\mathrm{n})(2+\mathrm{n})(3+\mathrm{n}) \mathrm{H}_{\mathrm{n}}\right\}\right\}
\end{aligned}
$$

The correctness of these solutions for $n \geq 0$ can be verified as sketched in Remark 3 .

Combining the solutions gives the closed form evaluation of $T_{n}=$ mySum2, namely

$\operatorname{In}[29]:=$ FindLinearCombination [recSol2, mySum2, 2, MinInitialValue $\rightarrow$ 0]

Out[29] $=1-9 n-9 n^{2}-2 n^{3}+2(1+n)(2+n)(3+n) H_{n}-(-1)^{n}$

which finally shows that $(2)$ holds for all $n \geq 0$.

\section{Conclusions}

In this survey article we illustrated how closed form evaluations of a very general class of definite multisums can be discovered with the summation package Sigma following the definite summation spiral. As example, we derived and proved the closed form evaluations of $S_{n}^{(1)}$ and $S_{n}^{(2)}$ from [FK00] purely algorithmically with computer algebra methods. For these computations the user is completely dispensed from working explicitly in difference fields or rings; instead one can work conveniently in terms of usual sum and product expressions.

\section{Acknowledgements}

I would like to thank Christian Krattenthaler for his valuable comments. 


\section{References}

[AP94] S. A. Abramov and M. Petkovšek. D'Alembertian solutions of linear differential and difference equations. In J. von zur Gathen, editor, Proc. ISSAC'94, pages 169-174. ACM Press, Baltimore, 1994.

[APP98] S. A. Abramov, P. Paule, and M. Petkovšek. $q$-Hypergeometric solutions of $q$-difference equations. Discrete Math., 180(1-3):3-22, 1998.

[Bro00] M. Bronstein. On solutions of linear ordinary difference equations in their coefficient field. J. Symbolic Comput., 29(6):841-877, June 2000.

[CD04] W. Chu and L. De Donno. Hypergeometric series and harmonic number identities. Preprint, 2004.

[Chy00] F. Chyzak. An extension of Zeilberger's fast algorithm to general holonomic functions. Discrete Math., 217:115-134, 2000.

[Coh65] R. M. Cohn. Difference Algebra. Interscience Publishers, John Wiley \& Sons, 1965.

[CS98] F. Chyzak and B. Salvy. Non-commutative elimination in ore algebras proves multivariate identities. J. Symbolic Comput., 26(2):187-227, 1998.

[DPSW04a] K. Driver, H. Prodinger, C. Schneider, and A. Weideman. Padé approximations to the logarithm II: Identities, recurrences, and symbolic computation. To appear in Ramanujan Journal, 2004.

[DPSW04b] K. Driver, H. Prodinger, C. Schneider, and A. Weideman. Padé approximations to the logarithm III: Alternative methods and additional results. To Appear in Ramanujan Journal, 2004.

[FK00] M. Fulmek and C. Krattenthaler. The number of rhombus tilings of a symmetric hexagon which contains a fixed rhombus on the symmetric axis, II. European J. Combin., 21(5):601640, 2000.

[Gos78] R. W. Gosper. Decision procedures for indefinite hypergeometric summation. Proc. Nat. Acad. Sci. U.S.A., 75:40-42, 1978.

[HS99] P. A. Hendriks and M. F. Singer. Solving difference equations in finite terms. J. Symbolic Comput., 27(3):239-259, 1999.

[Kar81] M. Karr. Summation in finite terms. J. ACM, 28:305-350, 1981.

[Kar85] M. Karr. Theory of summation in finite terms. J. Symbolic Comput., 1:303-315, 1985.

[KR04] C. Krattenthaler and T. Rivoal. Hypergéométrie et fonction zêta de Riemann. Preprint, 2004.

[Ma196] C. Mallinger. Algorithmic manipulations and transformations of univariate holonomic functions and sequences. Master's thesis, RISC, J. Kepler University, Linz, August 1996. 
[Pet92] M. Petkovšek. Hypergeometric solutions of linear recurrences with polynomial coefficients. J. Symbolic Comput., 14(2-3):243-264, 1992.

[PR97] P. Paule and A. Riese. A Mathematica q-analogue of Zeilberger's algorithm based on an algebraically motivated aproach to $q$-hypergeometric telescoping. In M. Ismail and M. Rahman, editors, Special Functions, q-Series and Related Topics, volume 14, pages 179-210. Fields Institute Toronto, AMS, 1997.

[PS95a] P. Paule and M. Schorn. A Mathematica version of Zeilberger's algorithm for proving binomial coefficient identities. J. Symbolic Comput., 20(5-6):673-698, 1995.

[PS95b] P. Paule and V. Strehl. Symbolic summation - some recent developments. In J. Fleischer et al., editor, Computer Algebra in Science and Engineering - Algorithms, Systems, and Applications, pages 138-162. World Scientific, Singapore, 1995.

[PS03] P. Paule and C. Schneider. Computer proofs of a new family of harmonic number identities. Adv. in Appl. Math., 31(2):359-378, 2003.

[PWZ96] M. Petkovšek, H. S. Wilf, and D. Zeilberger. A = B. A. K. Peters, Wellesley, MA, 1996.

[Rie03] A. Riese. qMultisum - A package for proving $q$-hypergeometric multiple summation identities. J. Symbolic Comput., 35:349-377, 2003.

[Ris69] R. Risch. The problem of integration in finite terms. Trans. Amer. Math. Soc., 139:167-189, 1969.

[Ris70] R. Risch. The solution to the problem of integration in finite terms. Bull. Amer. Math. Soc., 76:605-608, 1970.

[Sch00] C. Schneider. An implementation of Karr's summation algorithm in Mathematica. Sém. Lothar. Combin., S43b:1-10, 2000.

[Sch01] C. Schneider. Symbolic summation in difference fields. Technical Report 01-17, RISC-Linz, J. Kepler University, November 2001. PhD Thesis.

[Sch02a] C. Schneider. Degree bounds to find polynomial solutions of parameterized linear difference equations in $\Pi \Sigma$-fields. SFB-Report 02-21, J. Kepler University, Linz, November 2002.

[Sch02b] C. Schneider. Solving parameterized linear difference equations in $\Pi \Sigma$-fields. SFB-Report 02-19, J. Kepler University, Linz, November 2002.

[Sch04a] C. Schneider. A collection of denominator bounds to solve parameterized linear difference equations in $\Pi \Sigma$-extensions. To appear in SYNASC 2004, 2004.

[Sch04b] C. Schneider. Product representations in $\Pi \Sigma$-fields. To appear in Annals of Combinatorics, 2004.

[Sch04c] C. Schneider. Symbolic summation with single-nested sum extensions. Proc. ISSAC'O4, pages 282-289, 2004. 
[SZ94] B. Salvy and P. Zimmermann. Gfun: A package for the manipulation of generating and holonomic functions in one variable. ACM Trans. Math. Software, 20:163-177, 1994.

[vH98] M. van Hoeij. Rational solutions of linear difference equations. In O. Gloor, editor, Proc. ISSAC'98, pages 120-123. ACM Press, 1998.

[Weg97] K. Wegschaider. Computer generated proofs of binomial multi-sum identities. Diploma thesis, RISC Linz, Johannes Kepler University, May 1997.

[Zei90] D. Zeilberger. A fast algorithm for proving terminating hypergeometric identities. Discrete Math., 80(2):207-211, 1990. 